\title{
A regularity property for Schrödinger equations on bounded domains
}

\author{
Jean-Pierre Puel*
}

October 28, 2011

\begin{abstract}
We give a regularity result for the free Schrödinger equations set in a bounded domain of $\mathbb{R}^{N}$ which extends the 1-dimensional result proved in [2] with different arguments. We also give other equivalent results, for example, for the free Schrödinger equation, if the initial value is in $H_{0}^{1}(\Omega)$ and the right hand side $f$ can be decomposed in $f=g+h$ where $g \in L^{1}\left(0, T ; H_{0}^{1}(\Omega)\right)$ and $h \in L^{2}\left(0, T ; L^{2}(\Omega)\right), \Delta h=0$ and $h_{/ \Gamma} \in L^{2}\left(0, T ; L^{2}(\Gamma)\right)$, then the solution is in $C\left([0, T] ; H_{0}^{1}(\Omega)\right)$. This obviously contains the case $f \in L^{2}\left(0, T ; H^{1}(\Omega)\right)$. This result is essential for controllability purposes in the 1-dimensional case as shown in [2] and might be interesting for the $N$-dimensional case where the controllability problem is open.
\end{abstract}

\section{Introduction}

Let us consider the free Schrödinger equation (without potential) with Dirichlet boundary conditions on a bounded open domain $\Omega$ of $\mathbb{R}^{N}$ with boundary $\Gamma$ and on a time interval $(0, T)$ with $T>0$.

$$
\begin{aligned}
& i \frac{\partial y}{\partial t}+\Delta y=f \text { in } \Omega \times(0, T), \\
& y=0 \text { on } \Gamma \times(0, T), \\
& y(0)=y_{0} \text { in } \Omega .
\end{aligned}
$$

It is well known, from the properties of the Schrödinger group (see for example [6] or $[3])$, that when $y_{0} \in L^{2}(\Omega)$ and $f \in L^{1}\left(0, T ; L^{2}(\Omega)\right)$ then this equation has a unique

${ }^{*}$ Ikerbasque \& Basque Center for Applied Mathematics Bizkaia Technology Park, Building 500 E-48160 Derio Basque Country Spain and Laboratoire de Mathématiques de Versailles, Université de Versailles St Quentin, email : jppuel@bcamath.org. Part of this work was supported by the Visiting Fellow program of FP7-246775 NUMERIWAVES Grant. 
solution $y \in C\left([0, T] ; L^{2}(\Omega)\right)$. Moreover, if we denote by $\Delta$ the Laplace operator with Dirichlet boundary conditions, when $y_{0} \in \mathcal{D}\left(\Delta^{\frac{s}{2}}\right)$ and $f \in L^{1}\left(0, T ; \mathcal{D}\left(\Delta^{\frac{s}{2}}\right)\right)$ with $s \geq 0$, then $y \in C\left([0, T] ; \mathcal{D}\left(\Delta^{\frac{s}{2}}\right)\right)$.

On the other hand it is commonly accepted that the Schrödinger equation does not have any regularizing property.

Nevertheless, for purposes of exact controllability results, Karine Beauchard and Camille Laurent proved in [2] a regularity result in the 1-dimensional case $\Omega=] 0,1$, at least concerning the boundary conditions. Let us define the space

$$
H_{\Delta}(\Omega)=\left\{z \in H_{0}^{1}(\Omega), \Delta z \in H_{0}^{1}(\Omega)\right\} .
$$

Of course when $\Omega$ is regular enough we have

$$
H_{\Delta}(\Omega) \subset H^{3}(\Omega) .
$$

In [2] it is proved that when $y_{0} \in H_{\Delta}(0,1)$ and $f \in L^{2}\left(0, T ; H^{3}(0,1) \cap H_{0}^{1}(0,1)\right)$, then $y \in C\left([0, T] ; H_{\Delta}(0,1)\right)$. Notice the essential point that $f$ is not supposed to have values in $H_{\Delta}(0,1)$. In this article the proof makes essential use of explicit values of eigenvalues and eigenfunctions of $\frac{\partial^{2}}{\partial x^{2}}$ on $(0,1)$ and of fine harmonic analysis arguments. The result is essential for the study of controllability problems for Schrödinger equations. It enables the authors to establish the correct functional setting (the space $H_{\Delta}(0,1)$ ) when replacing $f(t, x)$ by $f(t, x)=u(t) \mu(x) y(t, x)$ with $u \in L^{2}(0, T)$ (control) and $\mu \in H^{3}(0,1)$ (potential profile). Using this regularity property, the authors have been able to simplify considerably and extend K.Beauchard's original proof of local exact controllability established in [1].

The goal of the present work is to extend this regularity result to the general case of a bounded open set $\Omega$ of $\mathbb{R}^{N}$. Of course in this case we cannot anymore use the explicit values for the eigenvalues and eigenfunctions of $\Delta$ and the proof will make use of arguments which, a priori, appear completely different.

Along the lines of the proof, we obtain several equivalent results which are worth being stated separately. All these results are given precisely in section 2 . Section 3 will contain the proof of the main theorem whereas the proofs of other results will be given in section 4 .

\section{Results}

We start with the result which motivated this work and extends the result in [2].

Theorem 2.1 Let $T$ be positive and $\Omega$ be a bounded open subset of $\mathbb{R}^{N}$ of class $C^{2, \alpha}$ with $\alpha>0$. We denote by $\Gamma$ the boundary of $\Omega$. For every $y_{0} \in H_{\Delta}(\Omega)$ and 
for every $f=g+h$ where

$$
g \in L^{1}\left(0, T ; H_{\Delta}(\Omega)\right)
$$

and

$$
h \in L^{2}\left(0, T ; H^{2}(\Omega) \cap H_{0}^{1}(\Omega)\right), \Delta^{2} h=0, \Delta h_{/ \Gamma} \in L^{2}\left(0, T ; L^{2}(\Gamma)\right),
$$

the solution $y$ of (1.1), (1.2), (1.3) satisfies

$$
y \in C\left([0, T] ; H_{\Delta}(\Omega)\right)
$$

and there exists $C>0$ independent of $y_{0}, g$ and $h$ such that

(2.8) $\|y\|_{C\left([0, T] ; H_{\Delta}(\Omega)\right)} \leq C\left(\left\|y_{0}\right\|_{H_{\Delta}(\Omega)}+\|g\|_{L^{1}\left(0, T ; H_{\Delta}(\Omega)\right)}+\left\|\Delta h_{/ \Gamma}\right\|_{L^{2}\left(0, T ; L^{2}(\Gamma)\right)}\right)$.

Remark 2.2 If $f \in L^{2}\left(0, T ; H^{3}(\Omega) \cap H_{0}^{1}(\Omega)\right)$, let us take $g$ such that for almost every $t \in(0, T)$

$$
\begin{aligned}
& \Delta^{2} g(t)=\Delta^{2} f(t) \text { in } \Omega, \\
& g=0 \text { on } \Gamma, \\
& \Delta g=0 \text { on } \Gamma .
\end{aligned}
$$

As $\Delta^{2} f \in L^{2}\left(0, T ; H^{-1}(\Omega)\right)$, this uniquely defines $g$ with $g \in L^{2}\left(0, T ; H_{\Delta}(\Omega)\right)$.

Let us now write $h=f-g$. Then $h \in L^{2}\left(0, T ; H^{2}(\Omega) \cap H_{0}^{1}(\Omega)\right), \Delta h \in L^{2}\left(0, T ; H^{1}(\Omega)\right)$ and

$$
\begin{aligned}
& \Delta^{2} h=0 \text { in } \Omega, \\
& h=0 \text { on } \Gamma, \\
& \Delta h_{/ \Gamma} \in L^{2}\left(0, T ; H^{\frac{1}{2}}(\Gamma)\right) .
\end{aligned}
$$

Therefore, Theorem 2.1 is indeed an extension of the result in [2].

In fact, Theorem 2.1 will be an immediate consequence of the following result.

Theorem 2.3 Let $T$ and $\Omega$ be as in the previous theorem. For every $y_{0} \in H_{0}^{1}(\Omega)$ and for every $f=g+h$ where

$$
g \in L^{1}\left(0, T ; H_{0}^{1}(\Omega)\right)
$$

and

$$
h \in L^{2}\left(0, T ; L^{2}(\Omega)\right), \Delta h=0, h_{/ \Gamma} \in L^{2}\left(0, T ; L^{2}(\Gamma)\right),
$$


the solution $y$ of (1.1), (1.2), (1.3) satisfies

$$
y \in C\left([0, T] ; H_{0}^{1}(\Omega)\right)
$$

and there exists $C>0$ independent of $y_{0}, g$ and $h$ such that

$$
\|y\|_{C\left([0, T] ; H_{0}^{1}(\Omega)\right)} \leq C\left(\left\|y_{0}\right\|_{H_{0}^{1}(\Omega)}+\|g\|_{L^{1}\left(0, T ; H_{0}^{1}(\Omega)\right)}+\left\|h_{/ \Gamma}\right\|_{L^{2}\left(0, T ; L^{2}(\Gamma)\right)}\right) .
$$

Remark 2.4 If $f \in L^{2}\left(0, T ; H^{1}(\Omega)\right)$, it can be decomposed as $f=g+h$ with $g \in$ $L^{2}\left(0, T ; H_{0}^{1}(\Omega)\right)$ and $h \in L^{2}\left(0, T ; H^{1}(\Omega)\right)$ with $\Delta h=0$ and so $h_{/ \Gamma} \in L^{2}\left(0, T ; H^{\frac{1}{2}}(\Gamma)\right)$.

Let us now consider the non homogeneous Schrödinger equation

$$
\begin{aligned}
& i \frac{\partial z}{\partial t}+\Delta z=f \text { in } \Omega \times(0, T), \\
& z=v \text { on } \Gamma \times(0, T), \\
& z(0)=z_{0} \text { in } \Omega .
\end{aligned}
$$

We will consider weak solutions of this problem in the sense of transposition which will be made precise in the next section. Theorem 2.3 and Theorem 2.1 will be consequences of the following result.

Theorem 2.5 Let $T$ and $\Omega$ be as in Theorem 2.1. For every $z_{0} \in H^{-1}(\Omega), v \in$ $L^{2}\left(0, T ; L^{2}(\Gamma)\right)$ and $f \in L^{1}\left(0, T ; H^{-1}(\Omega)\right)$, there exists a unique solution $z$ of (2.13), (2.14) and (2.15), in a sense which will be made precise below in Definition 3.2, satisfying

$$
z \in C\left([0, T] ; H^{-1}(\Omega)\right) .
$$

Moreover, there exists $C>0$ independent of $z_{0}, v$ and $f$ such that

$$
\|z\|_{C\left([0, T] ; H^{-1}(\Omega)\right)} \leq C\left(\left\|z_{0}\right\|_{H^{-1}(\Omega)}+\|f\|_{L^{1}\left(0, T ; H^{-1}(\Omega)\right)}+\|v\|_{L^{2}\left(0, T ; L^{2}(\Gamma)\right)}\right) .
$$

\section{Proof of Theorem 2.5}

First of all we have to give a precise definition for solutions of (2.13), (2.14) and (2.15). These solutions will be defined by transposition, following [4]. 


\subsection{Solutions by transposition}

The following results have already been obtained by Elaine Machtyngier in [5]. We give them again here for sake of completeness and also because we can slightly improve the required regularity.

In a first step, let us make some formal computations. Let us suppose that $z$ is a solution of $(2.13),(2.14)$ and $(2.15)$ with enough regularity. For every $\psi_{0} \in$ $H_{0}^{1}(\Omega)$ and $\varphi \in L^{1}\left(0, T ; H_{0}^{1}(\Omega)\right)$ we know that there exists a unique solution $\psi \in$ $C\left([0, T] ; H_{0}^{1}(\Omega)\right)$ of the following problem

$$
\begin{aligned}
& i \frac{\partial \psi}{\partial t}+\Delta \psi=\varphi \text { in } \Omega \times(0, T), \\
& \psi=0 \text { on } \Gamma \times(0, T), \\
& \psi(T)=\psi_{0} \text { in } \Omega .
\end{aligned}
$$

Let us take the scalar product of $(2.13)$ by $\psi$. We obtain formally

$\int_{0}^{T} \int_{\Omega} f \bar{\psi} d x d t=i\left(\int_{\Omega} z(T) \bar{\psi}_{0} d x-\int_{\Omega} z_{0} \bar{\psi}(0) d x\right)-\int_{0}^{T} \int_{\Gamma} v \frac{\partial \bar{\psi}}{\partial \nu} d \gamma d t+\int_{0}^{T} \int_{\Omega} z \bar{\varphi} d x d t$

where $\nu$ is the unit exterior normal vector to the boundary $\Gamma$.

Therefore, for every $\varphi \in L^{1}\left(0, T ; H_{0}^{1}(\Omega)\right)$, taking $\psi_{0}=0$ we have formally

$$
\int_{0}^{T} \int_{\Omega} z \bar{\varphi} d x d t=\int_{0}^{T} \int_{\Omega} f \bar{\psi} d x d t+i \int_{\Omega} z_{0} \bar{\psi}(0) d x+\int_{0}^{T} \int_{\Gamma} v \frac{\partial \bar{\psi}}{\partial \nu} d \gamma d t
$$

Notice that in the above formula, the integrals have to be understood as dualities and the term $\frac{\partial \psi}{\partial \nu}$ has to be defined in a correct way. This is the object of the following lemma which is a slight improvement of the result obtained in [5].

Lemma 3.1 ([5]) Let $\Omega$ be a bounded open subset of $\mathbb{R}^{N}$ of class $C^{2, \alpha}$ with $\alpha>0$ and let $T$ be positive. For every $\psi_{0} \in H_{0}^{1}(\Omega)$ and $\varphi \in L^{1}\left(0, T ; H_{0}^{1}(\Omega)\right)$ the solution $\psi$ of (3.18), (3.19), (3.20) satisfies

$$
\frac{\partial \psi}{\partial \nu} \in L^{2}\left(0, T ; L^{2}(\Gamma)\right)
$$

and the mapping

$$
\left(\psi_{0}, \varphi\right) \rightarrow \frac{\partial \psi}{\partial \nu}
$$

is linear continuous from $H_{0}^{1}(\Omega) \times L^{1}\left(0, T ; H_{0}^{1}(\Omega)\right)$ to $L^{2}\left(0, T ; L^{2}(\Gamma)\right)$. 
Proof.

First of all when the datas $\left(\psi_{0}, \varphi\right)$ are taken in a dense subset of regular functions, the solution $\psi$ is regular and $\frac{\partial \psi}{\partial \nu}$ makes perfect sense. Therefore it suffices to show that there exists a constant $C>0$ such that for every $\left(\psi_{0}, \varphi\right) \in C_{0}^{\infty}(\Omega) \times$ $C_{0}^{\infty}((0, T) \times \Omega)$ we have

$$
\left\|\frac{\partial \psi}{\partial \nu}\right\|_{L^{2}\left(0, T ; L^{2}(\Gamma)\right)} \leq C\left(\left\|\psi_{0}\right\|_{H_{0}^{1}(\Omega)}+\|\varphi\|_{L^{1}\left(0, T ; H_{0}^{1}(\Omega)\right)}\right) .
$$

In order to obtain this inequality, we use the multiplier method with a specific multiplier. Let $e_{1}$ be the positive unitary eigenfunction of $-\Delta$ on $\Omega$ associated with the first eigenvalue $\lambda_{1}$. From the regularity of $\Omega$ we know that $e_{1} \in C^{1}(\bar{\Omega})$ and from the strong maximum principle (see [7]) we know that there exists $\beta>0$ such that

$$
\forall x \in \Gamma,-\frac{\partial e_{1}}{\partial \nu}(x) \geq \beta>0
$$

We now take the mutiplier

$$
m=-\nabla e_{1}
$$

and multiply equation (3.18) by $m . \nabla \bar{\psi}$, then take the real part. We compute separately the different terms, taking into account that $\operatorname{div} m=-\Delta e_{1}=\lambda_{1} e_{1}$.

$$
\begin{aligned}
i \int_{0}^{T} & \int_{\Omega} \frac{\partial \psi}{\partial t} m \cdot \nabla \bar{\psi} d x d t=i \int_{\Omega}\left(\psi_{0} m \cdot \nabla \bar{\psi}_{0}-\psi(0) m \cdot \nabla \bar{\psi}(0)\right) d x-i \int_{0}^{T} \int_{\Omega} \psi m \cdot \nabla \frac{\partial \bar{\psi}}{\partial t} d x d t \\
\quad & i \int_{\Omega}\left(\psi_{0} m \cdot \nabla \bar{\psi}_{0}-\psi(0) m \cdot \nabla \bar{\psi}(0)\right) d x+i \int_{0}^{T} \int_{\Omega}\left(m \cdot \nabla \psi \frac{\partial \bar{\psi}}{\partial t}+\psi \frac{\partial \bar{\psi}}{\partial t} \operatorname{div} m\right) d x d t .
\end{aligned}
$$

Therefore

$$
\begin{gathered}
\operatorname{Re}\left(i \int_{0}^{T} \int_{\Omega} \frac{\partial \psi}{\partial t} m \cdot \nabla \bar{\psi} d x d t\right)= \\
\frac{i}{2} \int_{\Omega}\left(\psi_{0} m \cdot \nabla \bar{\psi}_{0}-\psi(0) m \cdot \nabla \bar{\psi}(0)\right) d x+\frac{\lambda_{1}}{2} \int_{0}^{T} \int_{\Omega} \psi\left(i \frac{\partial \bar{\psi}}{\partial t}\right) e_{1} d x d t= \\
\frac{i}{2} \int_{\Omega}\left(\psi_{0} m \cdot \nabla \bar{\psi}_{0}-\psi(0) m \cdot \nabla \bar{\psi}(0)\right) d x+\frac{\lambda_{1}}{2} \int_{0}^{T} \int_{\Omega} \psi(\Delta \bar{\psi}-\bar{\varphi}) e_{1} d x d t= \\
\frac{i}{2} \int_{\Omega}\left(\psi_{0} m \cdot \nabla \bar{\psi}_{0}-\psi(0) m \cdot \nabla \bar{\psi}(0)\right) d x+\frac{\lambda_{1}}{2} \int_{0}^{T} \int_{\Omega} \psi m \cdot \nabla \bar{\psi} d x d t \\
-\frac{\lambda_{1}}{2} \int_{0}^{T} \int_{\Omega}\left(|\nabla \psi|^{2}+\psi \bar{\varphi}\right) e_{1} d x d t .
\end{gathered}
$$


For the second term we have, noticing from (3.19) that on $\Gamma$ we have $\nabla \psi=(\nabla \psi \cdot \nu) \nu$

$$
\begin{array}{r}
\int_{0}^{T} \int_{\Omega} \Delta \psi m \cdot \nabla \bar{\psi} d x d t=\int_{0}^{T} \int_{\Gamma}(\nabla \psi \cdot \nu)(m \cdot \nabla \bar{\psi}) d \gamma d t \\
-\sum_{j, k=1}^{N} \int_{0}^{T} \int_{\Omega} \frac{\partial \psi}{\partial x_{j}} \frac{\partial m_{k}}{\partial x_{j}} \frac{\partial \bar{\psi}}{\partial x_{k}} d x d t-\sum_{j, k=1}^{N} \int_{0}^{T} \int_{\Omega} m_{k} \frac{\partial \psi}{\partial x_{j}} \frac{\partial^{2} \bar{\psi}}{\partial x_{k} \partial x_{j}} d x d t
\end{array}
$$

so that

$$
\begin{array}{r}
\operatorname{Re}\left(\int_{0}^{T} \int_{\Omega} \Delta \psi m \cdot \nabla \bar{\psi} d x d t\right)=\int_{0}^{T} \int_{\Gamma}|\nabla \psi \cdot \nu|^{2}(m . \nu) d \gamma d t \\
-\operatorname{Re}\left(\sum_{j, k=1}^{N} \int_{0}^{T} \int_{\Omega} \frac{\partial \psi}{\partial x_{j}} \frac{\partial m_{k}}{\partial x_{j}} \frac{\partial \bar{\psi}}{\partial x_{k}} d x d t\right)-\frac{1}{2} \sum_{k=1}^{N} \int_{0}^{T} \int_{\Omega} m_{k} \frac{\partial}{\partial x_{k}}\left(|\nabla \psi|^{2}\right) d x d t \\
=\frac{1}{2} \int_{0}^{T} \int_{\Gamma}|\nabla \psi \cdot \nu|^{2}(m . \nu) d \gamma d t-\operatorname{Re}\left(\sum_{j, k=1}^{N} \int_{0}^{T} \int_{\Omega} \frac{\partial \psi}{\partial x_{j}} \frac{\partial m_{k}}{\partial x_{j}} \frac{\partial \bar{\psi}}{\partial x_{k}} d x d t\right) \\
+\frac{\lambda_{1}}{2} \int_{0}^{T} \int_{\Omega}|\nabla \psi|^{2} e_{1} d x d t .
\end{array}
$$

Adding the two terms we obtain

$$
\begin{array}{r}
\frac{1}{2} \int_{0}^{T} \int_{\Gamma}|\nabla \psi \cdot \nu|^{2}(m . \nu) d \gamma d t=\operatorname{Re} \int_{0}^{T} \int_{\Omega} \varphi m \cdot \nabla \bar{\psi} d x d t-\frac{i}{2} \int_{\Omega}\left(\psi_{0} m \cdot \nabla \bar{\psi}_{0}-\psi(0) m \cdot \nabla \bar{\psi}(0)\right) d x \\
-\frac{\lambda_{1}}{2} \int_{0}^{T} \int_{\Omega}\left(\psi m \cdot \nabla \bar{\psi}+\psi \bar{\varphi} e_{1}\right) d x d t+\operatorname{Re}\left(\sum_{j, k=1}^{N} \int_{0}^{T} \int_{\Omega} \frac{\partial \psi}{\partial x_{j}} \frac{\partial m_{k}}{\partial x_{j}} \frac{\partial \bar{\psi}}{\partial x_{k}} d x d t\right) .
\end{array}
$$

From (3.24) and the standard estimates on solutions of (3.18), (3.19), (3.20), we then obtain with a constant $C$ independent of $\psi_{0}$ and $\varphi$

$$
\int_{0}^{T} \int_{\Gamma}|\nabla \psi \cdot \nu|^{2} d \gamma d t \leq C\left(\left\|\psi_{0}\right\|_{H_{0}^{1}(\Omega)}^{2}+\|\varphi\|_{L^{1}\left(0, T ; H_{0}^{1}(\Omega)\right)}^{2}\right) .
$$

This finishes the proof of Lemma 3.1.

We can now give a precise definition of solutions by transposition.

Definition 3.2 A function $z \in C\left([0, T] ; H^{-1}(\Omega)\right)$ is a (weak) solution to problem (2.13), (2.14), (2.15) if, for every $\varphi \in L^{1}\left(0, T ; H_{0}^{1}(\Omega)\right)$ and $\psi_{0}=0$ we have, if $\psi$ is the corresponding solution of (3.18), (3.19), (3.20),

$$
\begin{aligned}
\int_{0}^{T}<z, \bar{\varphi} & >_{H^{-1}(\Omega), H_{0}^{1}(\Omega)} d t=\int_{0}^{T}<f, \bar{\psi}>_{H^{-1}(\Omega), H_{0}^{1}(\Omega)} d t \\
& +i<z_{0}, \bar{\psi}(0)>_{H^{-1}(\Omega), H_{0}^{1}(\Omega)}+\int_{0}^{T} \int_{\Gamma} v \frac{\partial \bar{\psi}}{\partial \nu} d \gamma d t .
\end{aligned}
$$


For every $\varphi \in L^{1}\left(0, T ; H_{0}^{1}(\Omega)\right)$ let us define

$\mathcal{L}(\varphi)=\int_{0}^{T}<f, \bar{\psi}>_{H^{-1}(\Omega), H_{0}^{1}(\Omega)} d t+i<z_{0}, \bar{\psi}(0)>_{H^{-1}(\Omega), H_{0}^{1}(\Omega)}+\int_{0}^{T} \int_{\Gamma} v \frac{\partial \bar{\psi}}{\partial \nu} d \gamma d t$.

From Lemma 3.1 and classical results for Schrödinger equations (see for example [3]) the mapping

$$
\varphi \rightarrow \mathcal{L}(\varphi)
$$

is an antilinear continuous form on $L^{1}\left(0, T ; H_{0}^{1}(\Omega)\right)$. Therefore, there exists a unique element $z \in L^{\infty}\left(0, T ; H^{-1}(\Omega)\right)$ such that

$$
\forall \varphi \in L^{1}\left(0, T ; H_{0}^{1}(\Omega)\right), \int_{0}^{T}<z, \bar{\varphi}>_{H^{-1}(\Omega), H_{0}^{1}(\Omega)} d t=\mathcal{L}(\varphi) .
$$

Moreover we have

$$
\|z\|_{L^{\infty}\left(0, T ; H^{-1}(\Omega)\right)} \leq C\left(\left\|z_{0}\right\|_{H^{-1}(\Omega)}+\|f\|_{L^{1}\left(0, T ; H^{-1}(\Omega)\right)}+\|v\|_{L^{2}\left(0, T ; L^{2}(\Gamma)\right)}\right) .
$$

When the datas $z_{0}, f, v$ are taken in a dense subset of regular functions ( $C^{\infty}$ functions with compact support in space and time), it is well known that the solution $z$ is regular and we have $z \in C\left([0, T] ; H^{-1}(\Omega)\right)$ with the same estimate. Therefore, taking a sequence of regular datas $\left(z_{0}^{n}, f^{n}, v^{n}\right)$ converging to $\left(z_{0}, f, v\right)$ we have, denoting by $z^{n}$ the corresponding solution to (2.13), (2.14), (2.15),

$$
\begin{aligned}
\left\|z^{m}-z^{p}\right\|_{C\left([0, T] ; H^{-1}(\Omega)\right)} \leq C\left(\left\|z_{0}^{m}-z_{0}^{p}\right\|_{H^{-1}(\Omega)}\right. & +\left\|f^{m}-f^{p}\right\|_{L^{1}\left(0, T ; H^{-1}(\Omega)\right)} \\
& \left.+\left\|v^{m}-v^{p}\right\|_{L^{2}\left(0, T ; L^{2}(\Gamma)\right)}\right) .
\end{aligned}
$$

This shows that $z^{n}$ is a Cauchy sequence in $C\left([0, T] ; H^{-1}(\Omega)\right)$ and of course $z^{n}$ converges to $z$ so that $z \in C\left([0, T] ; H^{-1}(\Omega)\right)$. This finishes the proof of Theorem 2.5 .

\section{Proofs of Theorem 2.3 and of Theorem 2.1}

\subsection{Proof of Theorem 2.3}

Let us take

$$
\begin{gathered}
y_{0} \in C_{0}^{\infty}(\Omega), f=g+h, \\
g \in C_{0}^{\infty}\left(0, T ; C_{0}^{\infty}(\Omega)\right), h \in C_{0}^{\infty}\left(0, T ; W^{2, p}(\Omega)\right), \Delta h=0, h_{/ \Gamma} \in C_{0}^{\infty}\left(0, T ; C^{\infty}(\Gamma)\right) .
\end{gathered}
$$

This set of datas is dense in the general set of datas for Theorem 2.3. 
We then have, setting

$$
\begin{gathered}
z=\Delta y \\
i \frac{\partial z}{\partial t}+\Delta z=\Delta g \text { in } \Omega \times(0, T), \\
z=h / \Gamma \text { on } \Gamma \times(0, T) \\
z(0)=z_{0}=\Delta y_{0} \text { in } \Omega .
\end{gathered}
$$

Now from Theorem 2.5 we have

$$
\begin{aligned}
\|z\|_{C\left([0, T] ; H^{-1}(\Omega)\right)} \leq & C\left(\left\|z_{0}\right\|_{H^{-1}(\Omega)}+\|\Delta g\|_{L^{1}\left(0, T ; H^{-1}(\Omega)\right)}+\left\|h_{\Gamma \Gamma}\right\|_{L^{2}\left(0, T ; L^{2}(\Gamma)\right)}\right) \\
& \leq C\left(\left\|y_{0}\right\|_{H_{0}^{1}(\Omega)}+\|g\|_{L^{1}\left(0, T ; H_{0}^{1}(\Omega)\right)}+\left\|h_{/ \Gamma}\right\|_{L^{2}\left(0, T ; L^{2}(\Gamma)\right)}\right) .
\end{aligned}
$$

This implies

$$
\|y\|_{C\left([0, T] ; H_{0}^{1}(\Omega)\right)} \leq C\left(\left\|y_{0}\right\|_{H_{0}^{1}(\Omega)}+\|g\|_{L^{1}\left(0, T ; H_{0}^{1}(\Omega)\right)}+\left\|h_{/ \Gamma}\right\|_{L^{2}\left(0, T ; L^{2}(\Gamma)\right)}\right) .
$$

As in the previous section we can now use a density argument which shows immediately Theorem 2.3 .

\subsection{Proof of Theorem 2.1}

Let us now take

$$
y_{0} \in H_{\Delta}(\Omega), f=g+h,
$$

$g \in L^{1}\left(0, T ; H_{\Delta}(\Omega)\right), h \in L^{2}\left(0, T ; H^{2}(\Omega) \cap H_{0}^{1}(\Omega)\right), \Delta^{2} h=0, \Delta h_{\Gamma} \in L^{2}\left(0, T ; L^{2}(\Gamma)\right)$.

First of all it is clear that

$$
y \in C\left([0, T] ; H_{0}^{1}(\Omega)\right) .
$$

Let $z$ be solution of the following problem

$$
\begin{aligned}
& i \frac{\partial z}{\partial t}+\Delta z=\Delta g+\Delta h \text { in } \Omega \times(0, T), \\
& z=0 \text { on } \Gamma \times(0, T), \\
& z(0)=z_{0}=\Delta y_{0} \text { in } \Omega .
\end{aligned}
$$

From Theorem 2.3 we know that $z \in C\left([0, T] ; H_{0}^{1}(\Omega)\right)$, with

$$
\|z\|_{C\left([0, T] ; H_{0}^{1}(\Omega)\right)} \leq C\left(\left\|z_{0}\right\|_{H_{0}^{1}(\Omega)}+\|\Delta g\|_{L^{1}\left(0, T ; H_{0}^{1}(\Omega)\right)}+\left\|\Delta h_{/ \Gamma}\right\|_{L^{2}\left(0, T ; L^{2}(\Gamma)\right)}\right) .
$$


Let us now define $\tilde{y}$ by

$$
\Delta \tilde{y}=z, \tilde{y}_{/ \Gamma}=0
$$

we have

$$
\|\tilde{y}\|_{C\left([0, T] ; H_{\Delta}(\Omega)\right)} \leq C\left(\left\|y_{0}\right\|+\|g\|_{L^{1}\left(0, T ; H_{\Delta}(\Omega)\right)}+\left\|\Delta h_{/ \Gamma}\right\|_{L^{2}\left(0, T ; L^{2}(\Gamma)\right)}\right) .
$$

On the other hand it is immediate to see that

$$
\tilde{y}=y
$$

which finishes the proof of Theorem 2.1.

\section{References}

[1] K. Beauchard Local controllability of a 1D Schrödinger equation, J. Math. Pures et Appl., 84:851-956, July 2005.

[2] K. Beauchard, C. Laurent Local controllability of linear and nonlinear Schrödinger equations with bilinear control, to appear in J. Math. Pures et Appl.

[3] T. Cazenave, A. Haraux An Introduction to Semilinear Evolution Equations, Oxford University Press, 2006.

[4] J.-L. Lions, E. Magenes Problèmes aux limites non homogènes et applications, Vol I, Dunod, Paris, 1968.

[5] E. Machtyngier : Exact controllability for the Schrödinger equation, SIAM J. Control and Optim., 32 (1), 24-34, 1994.

[6] A. PAZy Semigroups of Linear Operators and Applications to Partial Differential Equations, Springer, 1983.

[7] M. Protter, H. Weinberger Maximum Principles in Differential Equations, Springer, 1984. 